\title{
COHERENT FORECASTING IN INTEGER-VALUED AR(1) MODELS WITH GEOMETRIC MARGINALS
}

\author{
Manik Awale, T. V. Ramanathan, Mohan Kale \\ Savitribai Phule Pune University
}

\begin{abstract}
This paper discusses the coherent forecasting in two types of integervalued geometric autoregressive time series models of order one, viz., Geometric Integer-valued Autoregressive (GINAR(1)) model and New Geometric Integer-valued Autoregressive (NGINAR(1)) model. GINAR(1) model uses binomial thinning for the process generation, whereas, NGINAR(1) uses negative binomial thinning. The k-step ahead conditional probability mass function and the corresponding probability generating functions are derived. It is observed that for higher order lags, the conditional mean, variance and the probability generating functions of these two processes are close to each other, whereas, for lower order lags, they differ. The coherent forecasting performance of these models is studied with the help of simulated and real data sets.
\end{abstract}

Key words: Binomial thinning, coherent forecasting, geometric integer- valued autoregressive models, negative binomial thinning.

\section{Introduction}

Integer-valued autoregressive models are extensively used for modeling the time series of counts that arises in many areas of natural and social sciences. Over dispersion is an inherent phenomenon of many such count time series data. This fact lead McKenzie (1986) to propose a geometric integer-valued autoregressive process of order one, viz., GINAR(1). The process generation in this case uses a binomial thinning mechanism, which was originally introduced by Steutal and Van Harn (1979). Mckenzie (1986) studied various probabilistic aspects of GINAR(1) model. Subsequently, after about two decades, Risti'c et al. (2009) have introduced a new geometric integer-valued autoregressive time series model of order one viz., NGINAR(1) with negative binomial thinning. These authors have obtained the k-step ahead probability generating function along with its mean and variance. Yule-Walker, conditional least squares and conditional maximum likelihood methods were used for the estimation of parameters of the proposed model. However, no comparisons were made with the GINAR(1) model in terms of its forecasting ability, which has the similar geometric marginal distribution.

Forecasting is an important issue (see Farrell et al. (2007) and Silva et al. (2009)), not exploited completely in GINAR(1) and NGINAR(1) models. Conditional mean, the traditional forecast, need not result as a count in the case of INAR models. However, the coherent forecasts do not violate this basic requirement. The coherent forecasts are usually obtained from the k-step ahead conditional distribution. The idea of coherent forecasting for INAR models was first proposed by Freeland and McCabe (2004). These authors have considered the coherent forecasting issues related to Poisson integer autoregressive model of order one proposed by Alosh and Alzaid (1987). Jung and Tremayne (2006) extended the idea of coherent forecasting to INAR(2) Poisson models and Bu and McCabe (2008) to 
INAR(p) models. Kim and Park (2010) studied the coherent forecasting in binomial autoregressive model of order p. Maiti et al. (2015) considered the coherent forecasting in count time series, using Box and Jenkins's AR(p) model. Recently, Maiti and Biswas (2015) have extended the coherent forecasting ideas to the over dispersed count time series data with GINAR(1) model. In their paper, the forecast values and measures have been compared with the Poisson INAR(1) forecasts. However, the over dispersed time series forecasts should be compared with forecasts obtained using over dispersed count time series models only. In this paper, we attempt to provide such a comparison of coherent forecasts using two geometric INAR(1) models. We also compare several other characteristics of these two integer-valued geometric time series models.

Count regression and time series models have abundant applications in epidemiology. Researchers have used various models for the analysis of epidemiological count data.O" zmen and Famoye (2007) used zero inflated count regression for modeling zoological data using zero inflated Poisson and generalized Poisson models. Lai (2005) used $\operatorname{AR}(1), \operatorname{ARMA}(1,1)$ and $\operatorname{ARIMA}(0,1,0)$ for the modeling of Severe Acute Respiratory Syndrome (SARS) epidemic data from China. Ngatchou-Wandji and Paris (2011) analyzed the annual incidence data of rhinitis, dermatitis and allergic asthma using zero-inflated count models and estimated the proportion of excess of zeros in future months. Kashikar et al. (2013) studied INAR model with structural breaks for analyzing the H1N1 data from Pune, India. Osisiogu and Nwosu (2015) considered the prediction of CD4 cell count of HIV/AIDS patients using a nonstationary Markov chain. In this paper, we dis- cuss two applications of geometric INAR models to epidemiological data related to measles and dengue from Germany.

The paper is organized as follows. In Section 2, we describe both the GINAR(1) and NGINAR(1) models and discuss some of their properties which are not reported earlier. Section 3 deals with the coherent forecasting of these models. A simulation study is reported in Section 4, which essentially compare these two models on various aspects, including coherent forecasting. Section 5 deals with the analysis of two real data sets using these models. Concluding remarks are presented in Section 6.

\section{Models with geometric marginals}

\subsection{Geometric INAR(1) model with binomial thinning}

The GINAR (1) process was first proposed by McKenzie (1986), who considered the binomial thinning to generate the process. The GINAR (1) process $\left\{X_{t}\right\}$ is given by the equation

$$
X_{t}=\phi \circ X_{t-1}+Z_{t}, t \geq 1,0 \leq \phi<1
$$

where, 'o' stands for the binomial thinning operator defined by

$$
\phi \circ \mathrm{X}=\sum_{i=1}^{X} W_{i}
$$

and $W_{i}^{\prime}$ s are independent and identically distributed (i.i.d.) Bernoulli $(\phi)$ random variables. It is assumed that $X_{0}$ is a geometric random variable with probability mass function (pmf)

$$
P\left[X_{0}=x\right]=(1-\theta) \theta^{x}, x \geq 0,0<\theta<1 .
$$


Here, $Z_{t}=U_{t} M_{t}, \forall t$, with Ut independent of $M_{t}, P\left[U_{t}=0\right]=\phi=1-P\left[U_{t}=1\right]$, and $\left\{M_{t}\right\}$ an i.i.d. geometric sequence with $P\left[M_{t}=j\right]=(1-\theta) \theta^{j}, j \geq 0$. Then, the marginal distribution of $\left\{X_{t}\right\}$ is given by

$$
P\left[X_{t}=x\right]=(1-\theta) \theta^{x}, x \geq 0,0<\theta<1 .
$$

The probability distribution of $Z_{t}$ is

$$
P\left(Z_{t}=z\right)=\left\{\begin{array}{r}
(1-\theta+\phi \theta), \text { if } z=0 \\
(1-\theta)(1-\phi) \theta^{z}, \text { if } z \geq 1
\end{array}\right.
$$

The marginal mean, variance and probability generating function (pgf) of $\left\{X_{t}\right\}$ are $E\left(X_{t}\right)$ $=\theta /(1-\theta), \mathrm{V}\left(X_{t}\right)=\theta /(1-\theta)^{2}$ and $\Phi X_{t}(s)=(1-\theta) /(1-s \theta)$ respectively. The conditional mean and the variance are

and

$$
\mathrm{E}\left(X_{t} \mid X_{t-1}\right)=\emptyset X_{t-1}+(1-\phi) \frac{\theta}{1-\theta}
$$

$$
\mathrm{V}\left(X_{t} \mid X_{t-1}\right)=\phi(1-\phi) X_{t-1}+(1-\phi) \theta(1+\phi \theta) \frac{1}{(1-\theta)^{2}}
$$

The conditional pmf is given by

$$
\begin{aligned}
\mathrm{P}\left(X_{t}\right. & \left.=\mathrm{y} \mid X_{t-1}=\mathrm{x}\right) \\
& =\left\{\begin{array}{c}
(1-\theta) \theta^{y-x} \sum_{r=0}^{y}\left(\begin{array}{l}
x \\
r
\end{array}\right) \theta^{x-r} \phi^{r}(1-\emptyset)^{x-r+1}+\left(\begin{array}{l}
x \\
y
\end{array}\right) \phi^{y+1}(1-\phi)^{x-y} \\
(1-\theta) \theta^{y-x}(1-\emptyset)\{\phi+(1-\phi) \theta\}^{x}, \text { if } y>x
\end{array}, \text { if } y \leq x\right.
\end{aligned}
$$

The k-step ahead conditional pgf of the process can be derived as,

$$
\begin{aligned}
\Phi_{X_{t+k} \mid X_{t}}(s)=E\left(E\left(s^{\phi \circ X_{t+k-1+Z}}\right) \mid X_{t}\right)=\Phi_{Z}(s) E\left(\left(\Phi_{W}(s)^{X_{t+k-1}} \mid X_{t}\right)\right. & \\
& =\prod_{i=0}^{k-1} \Phi_{Z}\left(\Phi_{W}^{(i)}(s)\right)\left(\Phi_{W}^{(k)}(s)\right)^{X_{t}}
\end{aligned}
$$

But,

$$
\Phi_{W}^{(k)}(s)=\Phi_{W} \Phi_{W}^{(k-1)}(s) \text { and } \Phi_{W}^{(0)}(s)=s
$$

Therefore using (1),

Hence the k-step ahead conditional pgf becomes,

$$
\Phi_{Z}(s)=\Phi_{X}(s)\left(\Phi_{X}\left(\Phi_{W}(s)\right)\right)^{-1}
$$

$$
\Phi_{X_{t+k} \mid X_{t}}(s)=\Phi_{X}(s)\left(\Phi_{X}\left(\Phi_{W}^{(k)}(s)\right)\right)^{-1}\left(\Phi_{W}^{(k)}(s)\right)^{X_{t}}
$$


But, $\Phi_{w}^{(k)}(\mathrm{s})=1-\phi^{k}+\phi^{k} s$. Therefore,

$$
\Phi_{X_{t+k} \mid X_{t}}(s)=\Phi X(s)\left(\Phi_{X}\left(1-\emptyset^{k}+\emptyset^{k} s\right)\right)^{-1}\left(1-\emptyset^{k}+\emptyset^{k} s\right)^{X_{t}}
$$

The auto correlation function is given by $\rho_{k}=\phi^{k}$, for $\mathrm{k} \geq 0$. The $\mathrm{k}$-step ahead conditional pmf is

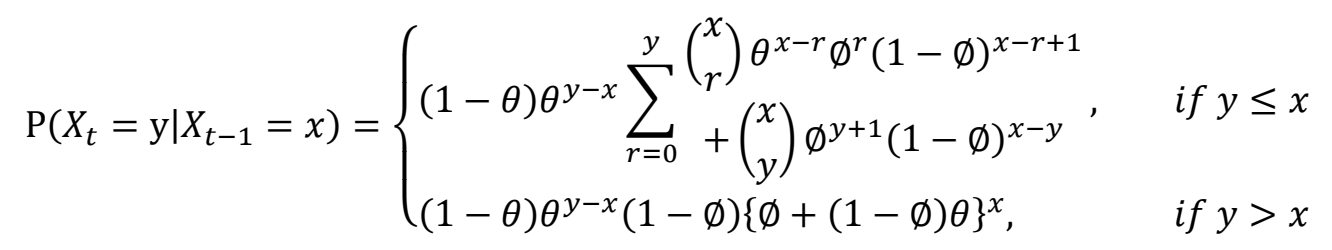

and the k-step ahead conditional mean and variance are respectively, and

$$
E\left(X_{t+k} \mid X_{t}\right)=\phi^{k} X_{t}+\left(1-\phi^{k}\right)
$$

and

$$
\mathrm{V}\left(X_{t+k} \mid X_{t}\right)=\emptyset^{k}\left(1-\emptyset^{k}\right) X_{t}+\frac{\emptyset}{1-\emptyset^{2}}\left(1-\emptyset^{k-1}-\emptyset^{k}+\emptyset^{2 k-1}\right) \mu_{z}+\frac{1-\emptyset^{2 k}}{1-\emptyset^{2}} \sigma_{z}^{2}
$$

where,

$$
\mu_{z}=\frac{\theta(1-\phi)}{1-\theta} \quad \text { and } \quad \sigma_{z}^{2}=\frac{(1-\phi) \theta(1+\phi \theta)}{(1-\theta)^{2}}
$$

are the mean and variance od $Z_{t}$. From the (4), (6) and (7), we observe that, as $k \rightarrow \infty$ the conditional pgf, mean and variance converge to the marginal pgf, mean and variance respectively.

\subsection{New Geometric INAR(1) model with negative binomial thinning}

The NGINAR(1) process $\left\{X_{t}\right\}$ introduced by Ristić et al. (2009) satisfies the equation

$$
\mathrm{X}_{t}=\alpha * \mathrm{X}_{t-1}+\epsilon_{t}, \quad t \geq 1
$$

where,

$$
\alpha * X=\sum_{i=1}^{X} W_{i}, \alpha \in[0,1),
$$

$\left\{W_{i}\right\}$ is sequence of i.i.d. random variables with

$$
P\left(W_{i}=w\right)=\alpha^{w} /(1+\alpha)^{w+1},
$$

$\epsilon_{t}$ is independent of $W_{i}$ and $X_{t-l}$ for all $l \geq 1$. The distribution of $\epsilon_{t}$ is, 


$$
\mathrm{P}\left(\epsilon_{t}=\mathrm{z}\right)=\left(1-\frac{\alpha \mu}{\mu-\alpha}\right) \frac{\mu^{z}}{(1+\mu)^{z+1}}+\frac{\alpha \mu}{\mu-\alpha} \frac{\alpha^{z}}{(1+\alpha)^{z+1}}, z=0,1,2, \ldots
$$

The process $\left\{X_{t}\right\}$ is stationary with

$$
P\left(X_{t}=x\right)=\mu^{x} /(1+\mu)^{x+1}, x=0,1,2, \ldots,
$$

where $\mu>0$. The unconditional mean, variance and pgf of $\left\{X_{t}\right\}$ are $E\left(X_{t}\right)=\mu, V\left(X_{t}\right)=\mu(1+$ $\mu)$ and $\Phi_{X t}(s)=1 /(1+\mu-s \mu)$ respectively. The Conditional pmf is,

$$
\mathrm{P}\left(X_{t}=\mathrm{y} \mid X_{t-1}=\mathrm{x}\right)= \begin{cases}\left(1-\frac{\alpha \mu}{\mu-\alpha}\right) \frac{\mu^{y}}{(1+\mu)^{y+1}}+\frac{\alpha \mu}{\mu-\alpha} \frac{\alpha^{y}}{(1+\alpha)^{y+1}}, & \text { if } x=0 \\
\frac{\mu \alpha^{y}}{(\mu-\alpha)(1+\alpha)^{y+x+1}}\left(\begin{array}{c}
y+x \\
y
\end{array}\right)+D, & \text { if } x \geq 1\end{cases}
$$

where,

$$
\mathrm{D}=\left(1-\frac{\alpha \mu}{(\mu-\alpha)}\right) \frac{\mu^{y}}{(1+\alpha)^{x}(1+\mu)^{y+1}} \sum_{r=0}^{y}\left(\begin{array}{c}
x+r-1 \\
x-1
\end{array}\right)\left(\frac{\alpha(1+\mu)}{\mu(1+\alpha)}\right)^{r}
$$

For obtaining k-step ahead conditional pgf and pmf, we proceed as follows.

We have $X_{t+1}=\alpha * X_{t}+\epsilon_{t+i}$. By repeated substitution

$$
X_{t+k}=\alpha * \alpha * \ldots \alpha * X_{t}+\left\{\alpha * . . \alpha * \epsilon_{t}+. .+\alpha * \epsilon_{t+k-1}+\epsilon_{t+k}\right\},
$$

which can be written as,

$$
X_{t+k}=\alpha^{(k)} * X_{t}+\sum_{j=0}^{k-1} \alpha^{(j)} * \epsilon_{t+k-j}
$$

Note that, $\alpha^{(i)} \neq \alpha^{i}, \alpha^{(i)}$ indicates the operation of negative binomial thinning $i$ times, but $\alpha^{(1)}=\alpha$ and $\alpha^{(0)}=1$. As $\epsilon_{t+i}$ is independent of $X \mathrm{t}$ for $i=1,2, \ldots$, the pgf of the two terms in the last expression can be found by taking the product of pgfs. Thus, the k-step ahead conditional pgf is,

$$
\begin{aligned}
\Phi_{X_{t+k} \mid X_{t}}(s)= & \Phi_{X}(s)\left\{\left(\frac{1-\alpha+\alpha\left(1-\alpha^{k-1}\right)(1-s)}{1-\alpha+\alpha\left(1-\alpha^{k}\right)(1-s)}\right)^{X_{t}}\right. \\
& \left.*\left(\frac{1-\alpha+\left(\alpha\left(1-\alpha^{k}\right)+(1-\alpha) \alpha^{k} \mu\right)(1-s)}{1-\alpha+\left(\alpha\left(1-\alpha^{k}\right)\right)(1-s)}\right)\right\},
\end{aligned}
$$

From (11), the k-step ahead pmf can be obtained as, 


$$
P\left(X_{t+k}=\mathrm{y} \mid X t=x\right)=\sum_{r=0}^{\mathrm{y}} P\left(\alpha^{(k)} * x=r\right) P\left(\sum_{j=0}^{k-1} \alpha^{(j)} * \epsilon_{t+k-j}=y-r\right),
$$

the probabilities in equation (13) are

$$
P\left(\alpha^{(k)} * \mathrm{x}=\mathrm{r}\right)= \begin{cases}A_{k}^{x}, & \text { if } r=0 \\
\frac{1}{r} \sum_{l=1}^{r} l\left(\begin{array}{l}
r \\
l
\end{array}\right) A_{k}^{x-1} B_{k}^{l} C_{k}^{r-1}\left(\begin{array}{l}
x \\
l
\end{array}\right), & \text { if } r \geq 1,\end{cases}
$$

$$
\begin{aligned}
\mathrm{P}\left(\sum_{j=0}^{k-1} \alpha^{(j)} * \varepsilon_{t+k-j}\right. & =\mathrm{y}-\mathrm{r})=\left(\frac{\left(\alpha^{k}-1\right)(\alpha-\mu+\alpha \mu)}{\alpha^{k+1}+\mu-\alpha(1+\mu)}\right) \frac{\mu^{y-r}}{(1+\mu)^{y-r+1}} \\
& +\frac{\alpha^{k} \mu(1-\alpha)}{\alpha^{k+1}+\mu-\alpha(1+\mu)}\left(\frac{1-\alpha}{1-\alpha^{k+1}}\right)\left(\frac{\alpha\left(1-\alpha^{k}\right)}{1-\alpha^{k+1}}\right)^{y-r}
\end{aligned}
$$

where, $A_{k}=\left(\frac{1-\alpha^{k}}{1-\alpha^{k+1}}\right), B_{k}=\left(\frac{\alpha^{k}(1-\alpha)^{2}}{\left(1-\alpha^{k+1}\right)^{2}}\right)$ and $C_{k}=\left(\frac{\alpha\left(1-\alpha^{k}\right)}{1-\alpha^{k+1}}\right)$

Using this, the k-step ahead conditional pmf can be obtained as

$$
\begin{gathered}
P\left(X_{t+k}=y \mid \mathrm{X}_{\mathrm{t}}=x\right) \\
P\left(\sum_{j=0}^{k-1} \alpha^{(j)} * \epsilon_{t+k-j}=y\right), \text { if } x=0 \\
P\left(\alpha^{(k)} * x=0\right) P\left(\sum_{j=0}^{k-1} \alpha^{(j)} * \epsilon_{t+k-j}=y\right) \\
+\sum_{r=1}^{y} P\left(\alpha^{(k)} * x=r\right) P\left(\sum_{j=0}^{k-1} \alpha^{(j)} * \epsilon_{t+k-j}=y-r\right), \text { if } x \geq 1 .
\end{gathered}
$$

The k-step ahead conditional mean and variance are,

$$
\mathrm{E}\left(X_{t+k} \mid X_{k}\right)=\alpha^{k} X_{t}+\frac{1-\alpha^{k}}{1-\alpha} \mu_{\epsilon}
$$

and 


$$
\begin{aligned}
& \mathrm{V}\left(X_{t+k} \mid X_{t}\right)=\frac{\alpha^{k}(1+\alpha)\left(1-\alpha^{k}\right)}{1-\alpha} X_{t}+\frac{1-\alpha^{2 k}}{1-\alpha^{2}} \sigma_{\epsilon}^{2}+ \\
& \left\{\frac{\alpha\left(1+\alpha+2 \mu_{\epsilon}\right)}{1-\alpha}\left(\frac{1-\alpha^{2 k}}{1-\alpha^{2}}\right)-\frac{1-\alpha^{k}}{1-\alpha} \alpha^{k-1}\right\} \mu_{\epsilon}+\left(\frac{1-\alpha^{2 k}}{1-\alpha^{2}}-\frac{1-2 \alpha^{k}+\alpha^{2 k}}{(1-\alpha)^{2}}\right) \mu_{\epsilon}^{2}
\end{aligned}
$$

where, $\mu_{\epsilon}=(1-\alpha) \mu$ and $\sigma_{\epsilon}^{2}=(1+\alpha) \mu((1+\mu)(1-\alpha)-\alpha)$ are the mean and variance of st. From (12), (16) and (17) we can see that the k-step ahead pgf, mean and variance converge to the unconditional pgf, mean and variance respectively as $k \rightarrow \infty$. The auto-correlation coefficient is $\rho_{k}=\alpha^{k}, k \geq 0$.

\subsection{Comparison of two geometric INAR models}

Ristić et al. (2009) have proposed the NGINAR(1) model after twenty three years of McKenzie's (1986) proposal of GINAR(1). Risti'c et al. (2009) have discussed the estimation of parameters and other properties in detail. However, McKenzie (1986) did not give much emphasis on estimation of the parameters. In a recent paper, Maity and Biswas (2015) studied the conditional least squares estimation in GINAR(1) model. An obvious question is about the preference between these two models, when the overdispersion is present in the count time series data. As per our knowledge, no attempt has been made to compare these two time series models in terms of their properties and forecasting ability. In this section we attempt to do this.

From the expressions of k-step ahead mean, variance and pgf of the two models, we see that they coincide as the lag $\mathrm{k} \rightarrow \infty$ and for $\mu=\theta /(1-\theta)$ and $\alpha=\phi$. Thus, it can be seen that these two models behave almost similarly after few lags for most of the parameter combinations, except when the parameter values are very small. Figure 1 represents the onestep ahead pmfs of GINAR(1) and NGINAR(1) models for same parameter combination, i.e., $\mu=\theta /(1-\theta)$ and $\alpha=\phi$. From this figure it can be seen that the conditional pmfs do differ for initial lags for same parameters. Figure 2 represents the k-step ahead conditional variance assuming same parameters for GINAR (1) and NGINAR(1). It can be seen that there is significant difference in the variability of the two processes for initial lags even after keeping parameter values same. Hence, this difference in the two processes with same marginals needs to be taken into account while modeling such data. 
(a)

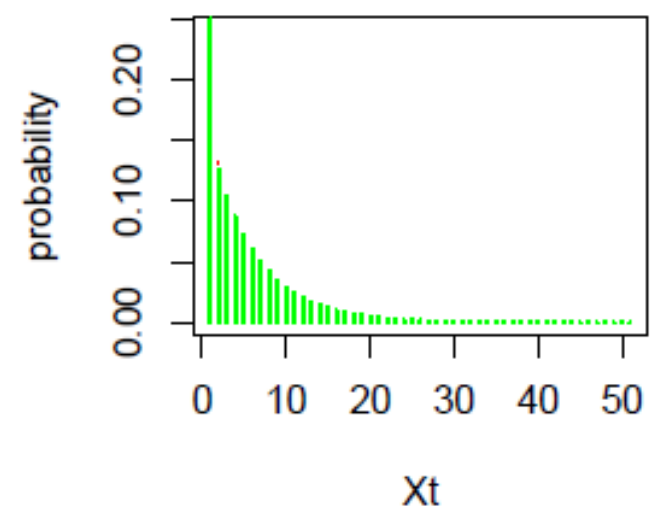

(c)

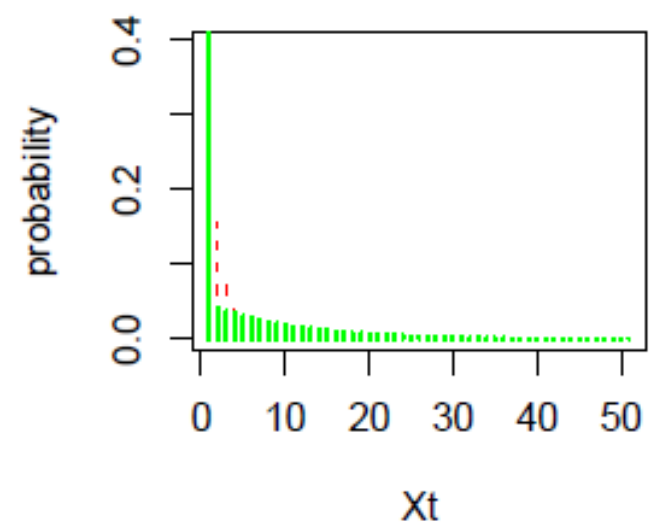

(b)

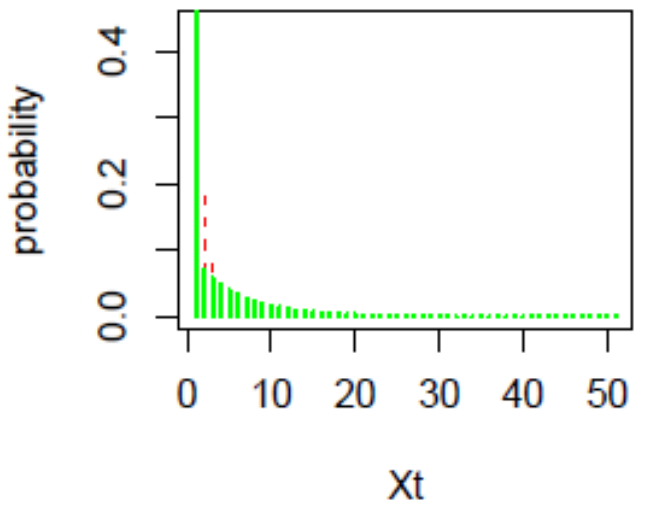

(d)

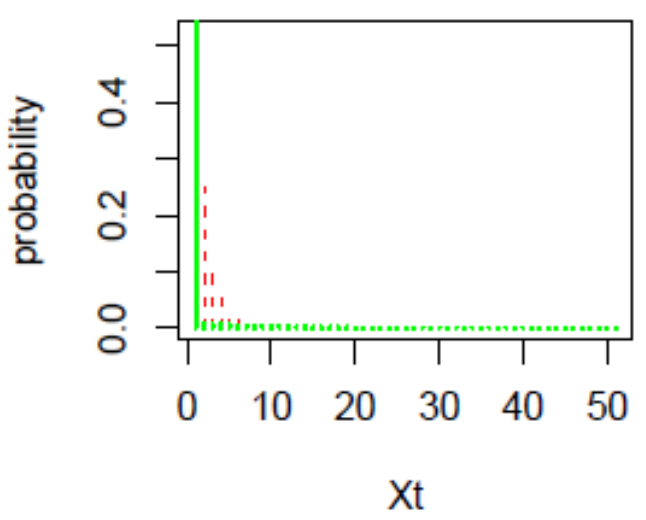

Figure 1: Comparison of conditional one step ahead pmf for various combinations of parameters, $(a)$ $\phi=0.1, \mu=5, n=50 ;(b) \phi=0.5, \mu=5$; (c) $\phi=0.5, \mu=10 ;(d) \phi=0.9, \mu=10$ and for $X_{t-l}=0$. Red dashed spikes indicate the NGINAR(1) model and Green spikes indicate GINAR(1) model. 

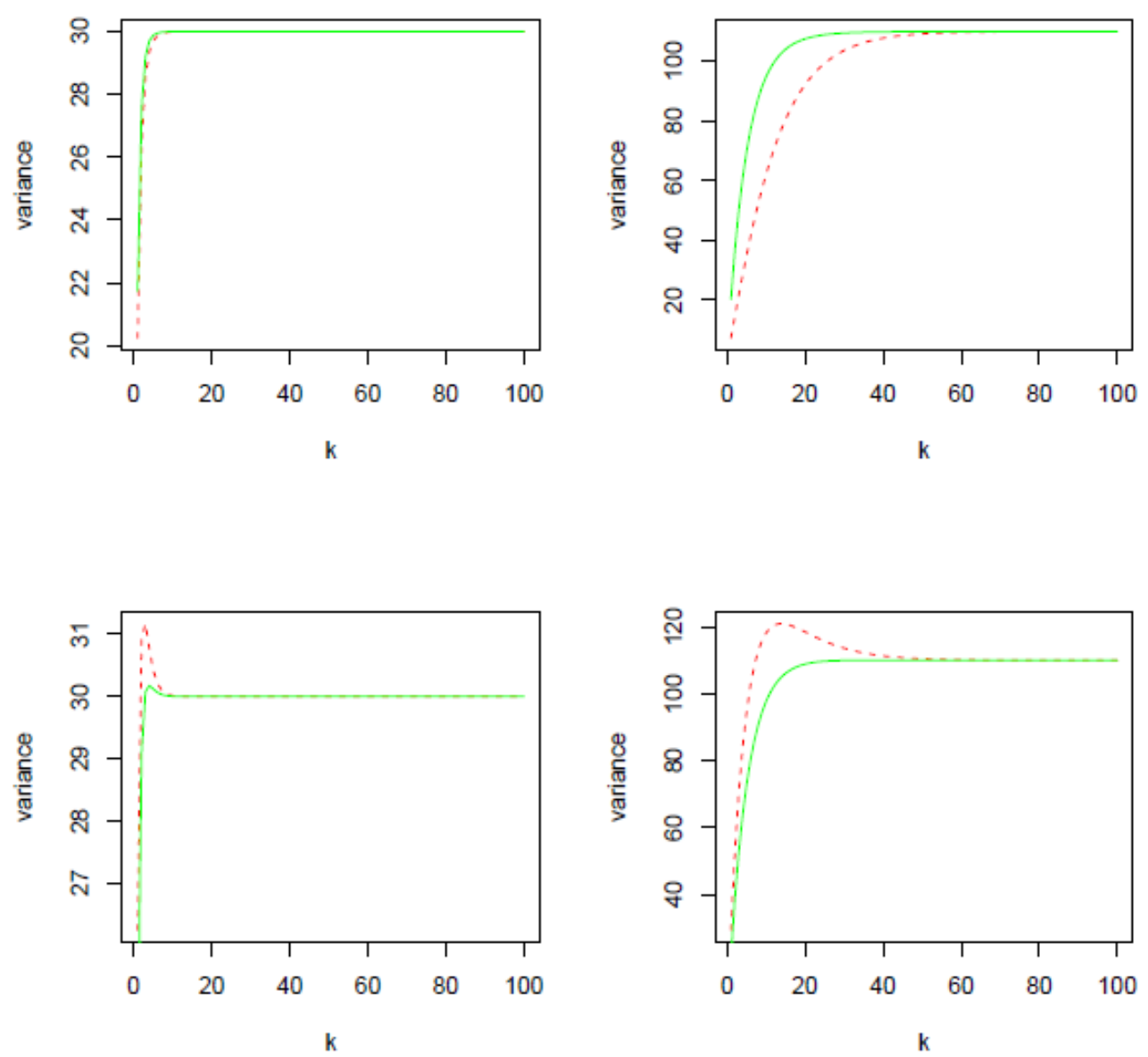

Figure 2: Comparison of k-step ahead conditional variances for various choice of parameters, $(a) \phi=$ $0.5, \mu=5, X_{t-1}=2$; (b) $\phi=0.9, \mu=10, X_{t-1}=2$; (c) $\phi=0.5, \mu=5, X_{t-1}=10$; (d) $\phi=0.9, \mu=10$, $X_{t-1}=15$. Red dashed line indicates the NGINAR(1) model and Green line indicates GINAR(1) model.

In order to study these two models, we have simulated 1000 data series, each of size 100 , 300,500 and 1000 from both the models with various combinations of the parameter values. The estimation results are presented in Table 1. The estimates are obtained using method of maximum likelihood. The last column of the Table 1 represents the percentage of the time the true model is selected using AIC criterion. If a series is generated using the NGINAR(1) model, then the AIC for NGINAR(1) model is expected to be smaller than that of the other model and vice-versa. From the last two columns of Table 1, it may be concluded that the correct model is selected majority of the times, except when the parameter values are very small. As the number of parameters in both models are same, BIC also gives same conclusion and hence not quoted here. 
Table 1: Two geometric INAR models: Estimation and model selection results

\begin{tabular}{|c|c|c|c|c|c|c|c|}
\hline \multirow[t]{2}{*}{ Original model } & \multirow[t]{2}{*}{ Sample size } & \multicolumn{2}{|c|}{ Parameters } & \multicolumn{2}{|c|}{ Mean estimates } & \multicolumn{2}{|c|}{ True model selection using AIC (\%) } \\
\hline & & $\alpha$ & $\mu$ & $\hat{\alpha}$ & $\hat{\mu}$ & NGINAR & GINAR \\
\hline \multirow{12}{*}{ NGINA R(1) } & 100 & 0.1 & 1 & 0.1013 & 0.9918 & 46.60 & 53.40 \\
\hline & & 0.3 & 3 & 0.2896 & 3.0231 & 63.40 & 36.60 \\
\hline & & 0.5 & 5 & 0.4496 & 4.9616 & 91.40 & 08.60 \\
\hline & 300 & 0.1 & 1 & 0.0980 & 1.0000 & 50.00 & 50.00 \\
\hline & & 0.3 & 3 & 0.3008 & 3.0081 & 80.20 & 19.80 \\
\hline & & 0.5 & 5 & 0.5009 & 4.9646 & 99.40 & 0.60 \\
\hline & 500 & 0.1 & 1 & 0.0973 & 0.9085 & 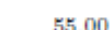 & 45,00 \\
\hline & & 0.3 & 3 & 0.3017 & 2.9806 & 87.20 & 12.80 \\
\hline & & 0.5 & 5 & 0.4991 & 5.0249 & 99.80 & 0.20 \\
\hline & 1000 & 0.1 & 1 & 0.1006 & 0.9998 & 54.20 & 45.80 \\
\hline & & 0.3 & 3 & 0.2995 & 2.9340 & 94.20 & 05.80 \\
\hline & & 0.5 & 5 & 0.4985 & 4.9991 & 100.0 & 0.00 \\
\hline & & $\phi$ & $\theta$ & $\phi$ & $\ddot{\theta}$ & & \\
\hline \multirow{12}{*}{ GINAR(1) } & 100 & 0.1 & 0.2 & 0.0962 & 0.1966 & 37.80 & 62.20 \\
\hline & & 0.5 & 0.4 & 0.4866 & 0.3913 & 01.20 & 98.80 \\
\hline & & 0.8 & 0.9 & 0.7984 & 0.8922 & 0.00 & 100.0 \\
\hline & 300 & 0.1 & 0.2 & 0.1002 & 0.2000 & 37.00 & 63.00 \\
\hline & & 0.5 & 0.4 & 0.4962 & 0.3993 & 0.00 & 100.0 \\
\hline & & 0.8 & 0.9 & 0.7997 & 0.8986 & 0.00 & 100.0 \\
\hline & 500 & 0.1 & 0.2 & 0.0976 & 0.1992 & 37.00 & 63.00 \\
\hline & & 0.5 & 0.4 & 0.4981 & 0.4000 & 0.00 & 1000 \\
\hline & & 0.8 & 0.9 & 0.7994 & 0.8983 & 0.00 & 100.0 \\
\hline & 1000 & 0.1 & 0.2 & 0.1005 & 0.2007 & 33.4 & 66.6 \\
\hline & & 0.5 & 0.4 & 0.4986 & 0.3978 & 0.00 & 100.0 \\
\hline & & 0.8 & 0.9 & 0.8002 & 0.9000 & 0.00 & 100.0 \\
\hline
\end{tabular}

\section{Remark 2.1:}

Suppose $P_{1}$ and $P_{2}$ denote the k-step transition probabilities of GINAR(1) and NGINAR(1) processes respectively. Then, the absolute difference between $\mathrm{P} 1$ and $\mathrm{P} 2$ is given by,

$$
\left|P_{l}\left(X_{t+k}=y \mid X_{\mathrm{t}}=x\right) \rightarrow P_{2}\left(X_{t+k}=y \mid X_{t}=x\right)\right|=d(x, y, k, \alpha, \mu)
$$

for all $\mathrm{x}$ and $\mathrm{y}, \alpha=\phi$ and $\theta=\mu /(1+\mu)$. Note that $\mathrm{d}(\cdot)$ is a bounded function, which tends to zero as $k \rightarrow \infty$. For example, when $x$ and $y$ are zero

$$
\mathrm{d}(0,0, k, \alpha, \mu)=\frac{\mu(1-\alpha) \alpha^{k+1}}{(1+k)\left(1-\alpha^{k+1}\right)} \rightarrow 0 \text { as } k \rightarrow \infty
$$

but, $\mathrm{d}(0,0,1, \alpha, \mu) f=0$. Similarly, for $x=1$ and $y=0$

$$
\mathrm{d}(1,0, k, \alpha, \mu)=\frac{\alpha^{k+1}\left(1-\alpha^{k}\right)\left(1-\alpha^{k+1}-\mu+2 \mu \alpha^{k}-\mu \alpha^{2 k+1}\right)}{\mu 1-\alpha^{k+1^{2}}}
$$

implies that $d(1,0, k, \alpha, \mu) \rightarrow 0$ as $k \rightarrow \infty$ but $\mathrm{d}(1,0,1, \alpha, \mu) f=0$ for $k=1$. Along with this it can be shown that as $k \rightarrow \infty$

and

$$
P_{l}\left(X_{t+k}=y \mid X_{\mathrm{t}}=x\right) \rightarrow P\left(X_{t+k}=y\right)=(1+\theta)^{y}, \quad \mathrm{y} \geq 0,
$$

$$
P_{2}\left(X_{t+k}=y \mid X_{\mathrm{t}}=x\right) \rightarrow P\left(X_{t+k}=y\right)=\mu_{y} /(1+\mu)^{y+1}, \quad \mathrm{y} \geq 0,
$$

where these two limiting pmfs are same under the condition $\theta=\mu /(1+\mu)$. From this, it is clear that the difference in the two conditional probabilities goes to zero as $\mathrm{k} \rightarrow \infty$ but it is not zero for small values of $k$. 


\section{Coherent forecasting}

In this section, we consider the coherent forecasting of GINAR(1) and NGINAR(1) models. The coherent forecasts are obtained using the median or mode of k-step ahead conditional probability distribution. The forecast accuracy is measured using measures such as Predictive Root Mean Square Error (PRMSE), Prediction Mean Absolute Error (PMAE) and Percentage of True Prediction(PTP). If the observed data set $\left\{X_{1}, \ldots, X_{n}, X_{n+1}, \ldots, X_{n+m}\right\}$ partitioned into two sets. The first $n$ observations are used for the estimation of the parameters of the model and rest m observations called the test set is used for the computation of these measures. These measures are defined as,

$$
\begin{aligned}
& \operatorname{PRMSE}(k)=\sqrt{\frac{1}{m-k+1}} \sum_{i=1}^{n+m-k}\left(X_{i+k}-\hat{X}_{i+k}^{\text {Mean }}\right) \\
& \operatorname{PMAE}(k)=\frac{1}{m-k+1} \sum_{i=1}^{n+m-k}\left|X_{i+k}-\hat{X}_{i+k}^{M e d}\right|
\end{aligned}
$$

and

$$
P T P=\frac{1}{m-k+1} \sum_{i=1}^{n+m-1} I\left(X_{i+k}=\hat{X}_{i+k}\right) \times 100 \%,
$$

where, I $(\cdot)$ is the indicator function. In PTP, we have used $\hat{X}_{t+k}=\hat{X}_{t+k}^{M e d}$ or $\hat{X}_{t+k}^{\text {Mode }}$ or the kstep ahead conditional mean, after rounding it to the nearest integer. $\hat{X}_{t+k}^{\text {Mode }}, \hat{X}_{t+k}^{\text {Median }}, \hat{X}_{t+k}^{\text {Mean }}$ are obtained from the k-step ahead conditional pmf.

The standard prediction intervals assume the predictive probability distribution to be symmetric . However, for these models, it is unimodal and positively skewed. Hence, we find the $100(1-\gamma) \%$ highest predictive probability interval (HPP) for $X_{t+k}$ as, $C_{k}=$ $\left(X_{L}, X_{U}\right)$, with $C_{k}=\left\{y: p_{k}(y \mid x) \geq K_{\gamma}\right\}$ and $K_{\gamma}$ is the largest number such that,

where

$$
\mathrm{P}\left(X_{L} \leq X_{t+k} \leq X_{U} \mid X_{t}=\mathrm{x}\right)=\sum_{y=X_{L}}^{X_{U}} p_{k(y \mid x) \geq(1-\gamma)},
$$

$$
p_{k}(y \mid x)=P\left(X_{t+k}=y \mid X_{t}=x\right) .
$$

Let $\hat{\alpha}_{t}$ and $\hat{\mu}_{t}$ be the maximum likelihood (ML) estimates of $\alpha$ and $\mu$ based on a sample of size $t$, then the ML estimate of the k-step ahead probability mass function is given by $p_{k}\left(y \mid X_{t} ; \hat{\alpha}_{t}, \hat{\mu}_{t}\right)$. Applying the $\delta$-method, one can derive the asymptotic distribution of this probability estimate, see Theorem 2 of Freeland \& McCabe (2004). Thus, the $95 \%$ confidence interval for $p_{k}\left(y \mid X_{t} ; \alpha_{0}, \mu_{0}\right)$ is, $p_{k}\left(y \mid X_{t} ; \hat{\alpha}_{t}, \hat{\mu}_{t}\right) \pm 2 \widehat{\sigma}_{k}\left(\mathrm{y} ; \hat{\alpha}_{t}, \hat{\mu}_{t}\right)$, where, $\sigma^{\wedge} \mathrm{k} 2\left(\mathrm{y} ; \hat{\alpha}_{t}, \hat{\mu}_{t}\right)$ is the estimated asymptotic variance of $p_{k}\left(y \mid X_{t} ; \hat{\alpha}_{t}, \hat{\mu}_{t}\right)$.

\section{Simulation study}

We have carried out three simulation studies. In the first study, we compare the two models with respect to their general properties and coherent forecasting ability. To achieve 
this, we have simulated 1000 series, each of size 700 from NGINAR(1) process with four sets of parameter values viz.; (a) $\alpha=0.6, \mu=2$, (b) $\alpha=0.6, \mu=2.5$, (c) $\alpha=0.4, \mu=1.5$ and (d) $\alpha=0.4, \mu=2$. The data are then divided into two parts, the first part is used for the estimation of parame- ters and the second to measure the forecast accuracy. Four hundred observations are used for parameter estimation and three hundred for computing the forecast accuracy measures. From Table 2 it can be observed that the values of the performance measures PRMSE and PMAE for the NGINAR(1) model are relatively lower for most of the

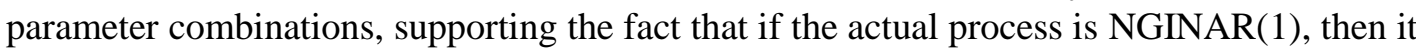
gives a better fit (less prediction error) than GINAR(1), even though the marginal distribution of the two processes is the same. It can be also seen that, as $\mathrm{k}$ increases, the values of the measures increases, indicating that the error in forecast increases as lag increases.

In the second simulation study, the performance of the conditional median or mode forecast is compared with the integer part of the conditional mean forecast.

Table 2: PRMSE and PMAE when the data are from NGINAR(1) model for $\mathrm{k}$-step ahead forecasts

\begin{tabular}{|c|c|c|c|c|c|c|c|c|}
\hline \multirow[t]{2}{*}{$k$} & \multicolumn{2}{|c|}{ GINAR(1) } & \multicolumn{2}{|c|}{ NGINAR(1) } & \multicolumn{2}{|c|}{ GINAR(1) } & \multicolumn{2}{|c|}{ NGINAR(1) } \\
\hline & PRMSE & PMAE & PRMSE & PMAE & PRMSE & PMAE & PRMSE & PMAE \\
\hline & \multicolumn{4}{|c|}{$\alpha=0.6, \mu=2$} & \multicolumn{4}{|c|}{$\alpha=0.6, \mu=2.5$} \\
\hline 1 & 2.0243 & 1.3410 & 1.9622 & 1.3085 & 2.4388 & 1.6257 & 2.3676 & 1.5676 \\
\hline 2 & 2.3403 & 1.5957 & 2.2889 & 1.5628 & 2.8250 & 1.9298 & 2.7623 & 1.8851 \\
\hline 3 & 2.4230 & 1.6533 & 2.3951 & 1.6347 & 2.9275 & 2.0249 & 2.8889 & 1.9947 \\
\hline 4 & 2.4444 & 1.6686 & 2.4309 & 1.6602 & 2.9557 & 2.0659 & 2.9333 & 2.0415 \\
\hline 5 & 2.4501 & 1.6712 & 2.4429 & 1.6717 & 2.9630 & 2.0818 & 2.9491 & 2.0624 \\
\hline & \multicolumn{4}{|c|}{$\alpha=0.4, \mu=1.5$} & \multicolumn{4}{|c|}{$\alpha=0.4, \mu=2$} \\
\hline 1 & 1.7860 & 1.1735 & 1.7764 & 1.1663 & 2.2556 & 1.4915 & 2.2319 & 1.4739 \\
\hline 2 & 1.9158 & 1.3014 & 1.9117 & 1.2986 & 2.4228 & 1.6448 & 2.4038 & 1.6285 \\
\hline 3 & 1.9334 & 1.3034 & 1.9324 & 1.3036 & 2.4458 & 1.6695 & 2.4306 & 1.6564 \\
\hline 4 & 1.9366 & 1.3039 & 1.9366 & 1.3040 & 2.4488 & 1.6714 & 2.4354 & 1.6610 \\
\hline 5 & 1.9375 & 1.3043 & 1.9377 & 1.3043 & 2.4485 & 1.6712 & 2.4363 & 1.6606 \\
\hline
\end{tabular}

Here we have used the same set up that of the first simulation study, except for the parameter values. The results of the study are reported in Table 3 for NGINAR(1) model. The values reported in Table 3 are the average PTP values over 1000 simulations, computed for each step. Here, we can see that the median and mode give better performance than the rounded Minimum Mean Square Error (MMSE) forecast. For the same data, the PTP values of mean, median and mode obtained using the GINAR(1) are presented in Table 4. In geometric case, the mode has high PTP value and hence it is preferred. Here, the PTP for mode using NGINAR(1) model is larger than GINAR(1) model.

In the third simulation study, we have simulated 305 observations from NGI- NAR(1) model, out of which 300 were used for the estimation and last five for out of sample forecast comparison. Based on the 300 observations we have computed the 100(1- $) \%$ HPP intervals discussed in Section 3. The test data are used to compare the prediction values such as mean, median and mode. These values are based on 1000 replications and are presented in Table 5 for NGINAR(1) model and in Table 6 for GINAR(1) model. All the values in the table are the averages over 1000 simulations. One can easily see that, the length of the HPP interval increases as the $\mathrm{k}$ increases. The average of the actual simulated values are re-ported in the 
second column. It may be noted that, the HPP intervals cover the average of the actual simulated values for all the parameter combinations. As NGINAR(1) model has more spread compared to GINAR(1), the HPP intervals

Table 3: PTP values in the case of NGINAR(1) model for k-step ahead forecasts when data are from NGINAR(1)

\begin{tabular}{|c|c|c|c|c|c|c|}
\hline \multirow[t]{2}{*}{$k$} & \multicolumn{3}{|c|}{ PTP } & \multicolumn{3}{|c|}{ PTP } \\
\hline & Mean & Median & Mode & Mean & Median & Mode \\
\hline \multicolumn{4}{|c|}{$\alpha=0.6, \mu=1.5$} & \multicolumn{3}{|c|}{$\alpha=0.6, \mu=2.5$} \\
\hline 1 & 23.18 & 39.86 & 42.66 & 18.29 & 30.09 & 32.76 \\
\hline 2 & 21.82 & 30.86 & 40.22 & 14.14 & 19.13 & 29.34 \\
\hline 3 & 21.23 & 24.03 & 39.99 & 13.03 & 18.59 & 28.70 \\
\hline 4 & 20.53 & 23.89 & 40.00 & 12.79 & 18.01 & 28.58 \\
\hline 5 & 20.00 & 23.98 & 40.00 & 12.65 & 17.45 & 28.55 \\
\hline \multicolumn{4}{|c|}{$\alpha=0.4, \mu=1.5$} & \multicolumn{3}{|c|}{$\alpha=0.4, \mu=2.5$} \\
\hline 1 & 22.07 & 37.92 & 41.71 & 14.84 & 21.90 & 31.66 \\
\hline 2 & 20.83 & 24.07 & 40.12 & 13.00 & 18.57 & 28.95 \\
\hline 3 & 19.97 & 24.01 & 40.04 & 12.73 & 17.66 & 28.61 \\
\hline 4 & 19.63 & 24.05 & 40.04 & 12.56 & 16.87 & 28.63 \\
\hline 5 & 19.46 & 24.05 & 40.03 & 12.53 & 16.68 & 28.63 \\
\hline
\end{tabular}

Table 4: PTP values in the case of GINAR(1) model for k-step ahead forecasts when data are from NGINAR(1)

\begin{tabular}{|c|c|c|c|c|c|c|}
\hline \multirow[t]{2}{*}{$k$} & \multicolumn{3}{|c|}{ PTP } & \multicolumn{3}{|c|}{ PTP } \\
\hline & Mean & Median & Mode & Mean & Median & Mode \\
\hline \multicolumn{4}{|c|}{$\alpha=0.6, \mu=1.5$} & \multicolumn{3}{|c|}{$\alpha=0.6, \mu=2.5$} \\
\hline 1 & 23.62 & 40.15 & 42.43 & 18.05 & 30.09 & 32.40 \\
\hline 2 & 22.71 & 31.25 & 40.13 & 14.04 & 20.08 & 29.10 \\
\hline 3 & 21.31 & 24.45 & 39.97 & 13.94 & 19.50 & 28.58 \\
\hline 4 & 22.19 & 24.12 & 39.99 & 13.91 & 19.20 & 28.58 \\
\hline 5 & 22.16 & 24.06 & 40.00 & 13.90 & 19.09 & 28.55 \\
\hline \multicolumn{4}{|c|}{$\alpha=0.4, \mu=1.5$} & \multicolumn{3}{|c|}{$\alpha=0.4, \mu=2.5$} \\
\hline 1 & 22.41 & 38.29 & 41.59 & 14.03 & 26.37 & 31.58 \\
\hline 2 & 21.07 & 24.37 & 40.06 & 13.36 & 19.11 & 28.83 \\
\hline 3 & 20.81 & 24.06 & 40.04 & 13.29 & 17.99 & 28.59 \\
\hline 4 & 20.71 & 24.05 & 40.04 & 13.26 & 17.63 & 28.63 \\
\hline 5 & 20.69 & 24.05 & 40.03 & 13.25 & 17.61 & 28.63 \\
\hline
\end{tabular}

computed using NGINAR(1) are wider than that using GINAR(1). We employed the same parameter combination and data for the both the cases. From Tables 5 and 6 it can be seen that the estimated mean, median and mode using the GI- NAR(1) model are smaller than the NGINAR(1), indicating that the NGINAR(1) model can accommodate larger counts than the GINAR(1).

Table 5: HPP intervals ( $80 \%$ ) for the NGINAR(1) model for simulated data with average forecasted values for different sets of parameters when data are from NGINAR(1) 


\begin{tabular}{|c|c|c|c|c|c|c|c|c|c|c|}
\hline \multirow[b]{2}{*}{$k$} & \multicolumn{5}{|c|}{$\alpha=0.4, \mu=1.5$} & \multicolumn{5}{|c|}{$\alpha=0.4, \mu=2.5$} \\
\hline & $\left(X_{L}, X_{U}\right)$ & $\bar{X}_{300+k}$ & mean & median & mode & $\left(X_{L}, X_{U}\right)$ & $\bar{X}_{300+k}$ & mean & median & mode \\
\hline 1 & {$[0.04,2.54)$} & 1.603 & 1.520 & 0.897 & 0.362 & {$[0.14,3.98)$} & 2.559 & 2.493 & 1.745 & 0.793 \\
\hline 2 & {$[0,2.61)$} & 1.575 & 1.515 & 1.052 & 0.032 & {$[0,4.24)$} & 2.526 & 2.497 & 1.515 & 0.171 \\
\hline 3 & {$[0,2.68)$} & 1.505 & 1.512 & 1.006 & 0.001 & {$[0,4.28)$} & 2.442 & 2.497 & 1.523 & 0.017 \\
\hline 4 & {$[0,2.72)$} & 1.500 & 1.510 & 1.002 & 0.000 & {$[0,4.26)$} & 2.432 & 2.497 & 1.589 & 0.000 \\
\hline \multirow[t]{2}{*}{5} & {$[0,2.74)$} & 1.460 & 1.510 & 1.000 & 0.000 & {$[0,4.26)$} & 2.510 & 2.497 & 1.620 & 0.000 \\
\hline & \multicolumn{5}{|c|}{$\alpha=0.6, \mu=1.5$} & \multicolumn{5}{|c|}{$\alpha=0.6, \mu=2.5$} \\
\hline 1 & {$[0.10,2.51)$} & 1.462 & 1.534 & 1.086 & 0.619 & {$[0.28,3.92)$} & 2.464 & 2.410 & 1.769 & 0.119 \\
\hline 2 & {$[0,2.71)$} & 1.475 & 1.522 & 0.933 & 0.118 & {$[0.03,4.06)$} & 2.439 & 2.440 & 1.698 & 0.388 \\
\hline 3 & {$[0,2.61)$} & 1.456 & 1.515 & 1.073 & 0.011 & {$[0,4.18)$} & 2.453 & 2.458 & 1.518 & 0.097 \\
\hline 4 & {$[0,2.62)$} & 1.508 & 1.511 & 1.026 & 0.000 & {$[0,4.24)$} & 2.579 & 2.469 & 1.484 & 0.021 \\
\hline 5 & {$[0,2.65)$} & 1.547 & 1.509 & 1.006 & 0.000 & {$[0,4.24)$} & 2.557 & 2.475 & 1.513 & 0.003 \\
\hline
\end{tabular}

\section{Real dataanalysis}

\subsection{Dengue data}

Consider the weekly data on Dengue cases during the year 2010 to 2015, from Berlin, available at https://survstat.rki.de. The data consist of 339 observations. From the descriptive statistics given in Table 7, note that the sample variance is larger than the sample mean, which leads to an over dispersed situation. Thus, the usual Poisson INAR model is not expected to give a good fit and hence geometric INAR models are preferred.

The AR(1) structure of the model can be justified from the partial autocorrelation function (PACF) plot in Figure 3. From Table 8, we see that the AIC for NGINAR(1) model is smaller than that of GINAR(1), and thus the data support the NGINAR(1) model. The forecast accuracy measures for NGINAR(1)

Table 6: HPP intervals (80\%) for the GINAR(1) model for simulated data with average forecasted values for different sets of parameters when data are from NGINAR(1)

\begin{tabular}{|c|c|c|c|c|c|c|c|c|c|c|}
\hline \multirow[b]{2}{*}{$k$} & \multicolumn{5}{|c|}{$\alpha=0.4, \mu=1.5$} & \multicolumn{5}{|c|}{$\alpha=0.4, \mu=2.5$} \\
\hline & $\left(X_{L}, X_{U}\right)$ & $X_{300+k}$ & mean & median & mode & $\left(X_{L}, X_{U}\right)$ & $X_{300+k}$ & mean & median & mode \\
\hline 1 & {$[0.04,2.47)$} & 1.489 & 1.473 & 0.797 & 0.436 & {$[0.15,4.00)$} & 2.346 & 2.436 & 1.383 & 0.819 \\
\hline 2 & {$[0,2.57)$} & 1.484 & 1.464 & 0.997 & 0.063 & {$[0,4.13)$} & 2.399 & 2.416 & 1.418 & 0.200 \\
\hline 3 & {$[0,2.63)$} & 1.537 & 1.462 & 1.000 & 0.004 & {$[0,4.11)$} & 2.575 & 2.410 & 1.468 & 0.028 \\
\hline 4 & {$[0,2.63)$} & 1.481 & 1.461 & 1.000 & 0.000 & {$[0,4.11)$} & 2.394 & 2.407 & 1.479 & 0.000 \\
\hline \multirow[t]{2}{*}{5} & {$[0,2.64)$} & 1.518 & 1.461 & 1.000 & 0.000 & {$[0,4.11)$} & 2.493 & 2.407 & 1.479 & 0.000 \\
\hline & \multicolumn{5}{|c|}{$\alpha=0.6, \mu=1.5$} & \multicolumn{5}{|c|}{$\alpha=0.6, \mu=2.5$} \\
\hline 1 & {$[0.10,2.26)$} & 1.571 & 1.410 & 0.862 & 0.598 & {$[0.28,3.64)$} & 2.518 & 2.346 & 1.424 & 1.050 \\
\hline 2 & {$[0,2.37)$} & 1.480 & 1.387 & 0.765 & 0.159 & {$[0.03,3.85)$} & 2.482 & 2.281 & 1.405 & 0.412 \\
\hline 3 & {$[0,2.39)$} & 1.455 & 1.379 & 0.976 & 0.017 & {$[0,3.86)$} & 2.621 & 2.254 & 1.284 & 0.121 \\
\hline 4 & {$[0,2.39)$} & 1.472 & 1.377 & 0.992 & 0.000 & {$[0,3.86)$} & 2.605 & 2.243 & 1.260 & 0.023 \\
\hline 5 & {$[0,2.40)$} & 1.542 & 1.376 & 0.995 & 0.000 & {$[0,3.87)$} & 2.688 & 2.238 & 1.233 & 0.001 \\
\hline
\end{tabular}

model are better than that of GINAR(1) model. The first 275 observations were used for the estimation of the parameters and remaining for testing, i.e., to compute the forecast measures. It can be seen that the NGINAR(1) model performs better than the GINAR(1), in terms of these measures. The point forecast of the probabilities for the Dengue data using NGINAR(1) model are given in Ta- ble 9. From this table, it can be observed that the transition probabilities from 0 to 1 are increasing slowly as lag increases and become steady for higher lags and are less than 
0.250. The transition probabilities from 0 to other values are decreasing for steps 1 to 5 , indicating that, in future, disease will remain under control. These probabilities are obtained for steps 1 to 5 and for values 0 to 5 . The probabilities of the values beyond 5 are not given, as they are very small and are not going to add much information. It can be observed that the median and mode are also 0 for all the steps of the forecast distribution, which indicates that, in future, the number of Dengue cases will be zero with high probability and one with moderate probability. The probability estimates reveal that there is nearly $90 \%$ chance that the number of cases in next step is less than or equal to 2 . We have obtained the $95 \%$ confidence intervals for the probabilities and they are given in Table 10. It can be concluded from Table 10 that the probability of 0 case in the next period lies between 0.517 to 0.656 and probability of 1 case in the next period lies between 0.210 and 0.236 with $95 \%$ confidence. From Table 9 we can see that the point estimate of probability for zero is around half and the probability is decreasing for higher values, implying that the chance of having value higher than one is quite less. Table 11 gives the HPP intervals (80\%) for the forecast values. These intervals are obtained for all the five-step ahead forecasts. It may be noted that the median and mode forecasts are better than the mean forecasts.

Time series plot
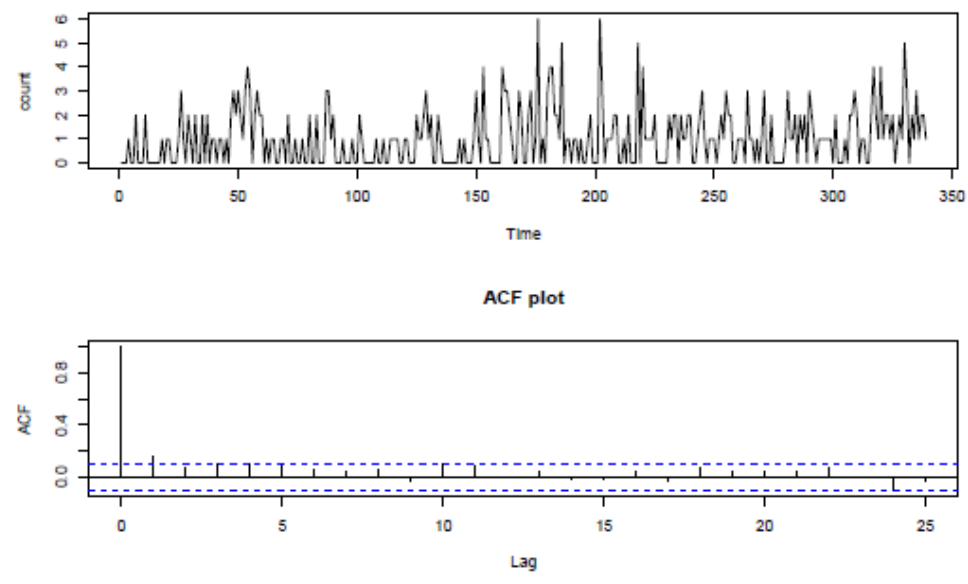

PACF plot

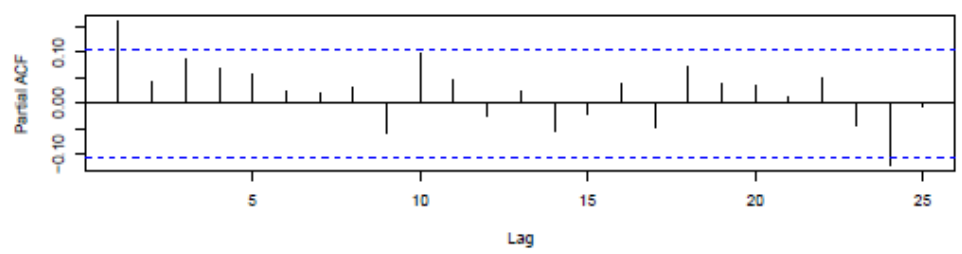

Figure 3: Time series, ACF and PACF plots of Dengue data

\subsection{Measles data}

These data consist of 234 observations on weekly cases of measles form the state Rhineland-Palatinate of Germany, during the period 2006 to 2010, the data are available from https://survstat.rki.de. The data appear to be clearly over dispersed from the descriptive statistics given in Table 12, and therefore the

Table 7: Descriptive statistics of Dengue data 


\begin{tabular}{cccccc}
\hline Minimum & Maximum & Median & Mode & Mean & Variance \\
\hline 0 & 6 & 1 & 0 & 1.035 & 1.3951 \\
\hline
\end{tabular}

Table 8: Predictive analysis of Dengue data

\begin{tabular}{|c|c|c|c|c|c|c|c|}
\hline Model & Estimated Values & AIC & $k$-step & PRMSE & PMAE & $\begin{array}{c}\text { PTP } \\
\text { (Median) }\end{array}$ & $\begin{array}{l}\text { PTP } \\
\text { (Mode) }\end{array}$ \\
\hline NGINAR(1) & $\begin{array}{l}\alpha=0.1773 \\
\mu=0.9487\end{array}$ & 747.771 & $\begin{array}{l}1 \\
2 \\
3 \\
4 \\
5\end{array}$ & $\begin{array}{l}1.163 \\
1.184 \\
1.193 \\
1.197 \\
1.201\end{array}$ & $\begin{array}{l}0.906 \\
1.079 \\
1.354 \\
1.377 \\
1.400\end{array}$ & $\begin{array}{l}37.50 \\
30.16 \\
24.19 \\
22.95 \\
21.67\end{array}$ & $\begin{array}{l}26.56 \\
25.40 \\
24.19 \\
22.95 \\
21.67\end{array}$ \\
\hline GINAR(1) & $\begin{array}{c}\phi=0.1200, \\
\theta=0.4867\end{array}$ & 749.5052 & $\begin{array}{l}1 \\
2 \\
3 \\
4 \\
5\end{array}$ & $\begin{array}{l}1.167 \\
1.187 \\
1.194 \\
1.197 \\
1.201\end{array}$ & $\begin{array}{l}1.109 \\
1.269 \\
1.354 \\
1.377 \\
1.400\end{array}$ & $\begin{array}{l}32.81 \\
26.98 \\
24.19 \\
22.95 \\
21.67\end{array}$ & $\begin{array}{l}26.56 \\
25.40 \\
24.19 \\
22.95 \\
21.67\end{array}$ \\
\hline
\end{tabular}

Table 9: Forecast probabilities for Dengue data using NGINAR(1) model

\begin{tabular}{cccccc}
\hline & \multicolumn{5}{c}{$k$} \\
\cline { 2 - 6 } & 1 & 2 & 3 & 4 & 5 \\
\hline$p_{k}(0 \mid 0)$ & 0.586 & 0.526 & 0.515 & 0.514 & 0.513 \\
$p_{k}(1 \mid 0)$ & 0.223 & 0.246 & 0.249 & 0.250 & 0.250 \\
$p_{k}(2 \mid 0)$ & 0.099 & 0.118 & 0.121 & 0.122 & 0.121 \\
$p_{k}(3 \mid 0)$ & 0.047 & 0.057 & 0.059 & 0.060 & 0.059 \\
$p_{k}(4 \mid 0)$ & 0.023 & 0.028 & 0.029 & 0.029 & 0.029 \\
$p_{k}(5 \mid 0)$ & 0.011 & 0.014 & 0.014 & 0.014 & 0.014 \\
\hline
\end{tabular}

Table 10: Confidence interval (95\%) for the forecast probabilities from Dengue data using NGINAR(1) model

\begin{tabular}{lccccc}
\hline & \multicolumn{5}{c}{$k$} \\
\cline { 2 - 6 } & 1 & 2 & 3 & 4 & 5 \\
\hline \multirow{2}{*}{$p_{k}(0 \mid 0)$} & $(0.517,0.656)$ & $(0.476,0.576)$ & $(0.466,0.565)$ & $(0.463,0.564)$ & $(0.463,0.564)$ \\
$p_{k}(1 \mid 0)$ & $(0.210,0.236)$ & $(0.240,0.251)$ & $(0.247,0.251)$ & $(0.248,0.251)$ & $(0.249,0.251)$ \\
$p_{k}(2 \mid 0)$ & $(0.077,0.122)$ & $(0.104,0.131)$ & $(0.108,0.134)$ & $(0.108,0.135)$ & $(0.108,0.135)$ \\
$p_{k}(3 \mid 0)$ & $(0.031,0.063)$ & $(0.045,0.069)$ & $(0.047,0.071)$ & $(0.047,0.072)$ & $(0.047,0.072)$ \\
$p_{k}(4 \mid 0)$ & $(0.013,0.032)$ & $(0.019,0.036)$ & $(0.020,0.038)$ & $(0.020,0.038)$ & $(0.020,0.038)$ \\
$p_{k}(5 \mid 0)$ & $(0.005,0.016)$ & $(0.007,0.018)$ & $(0.008,0.020)$ & $(0.008,0.020)$ & $(0.008,0.020)$ \\
\hline
\end{tabular}

Table 11: Prediction interval $(80 \%)$ with actual and forecast values using the mean, median and mode of the conditional distribution for Dengue data using NGINAR(1) model

\begin{tabular}{cccccc}
\hline$k$ & actual & $\left(X_{L}, X_{U}\right)$ & Mean & Median & Mode \\
\hline 1 & 0 & {$[0,2)$} & 0.7804 & 0 & 0 \\
2 & 0 & {$[0,3)$} & 0.9188 & 0 & 0 \\
3 & 0 & {$[0,3)$} & 0.9434 & 0 & 0 \\
4 & 0 & {$[0,3)$} & 0.9477 & 0 & 0 \\
5 & 1 & {$[0,3)$} & 0.9485 & 0 & 0
\end{tabular}

Poisson INAR(1) model is not a good choice. The AR(1) structure of the data can be concluded from the PACF plot in Figure 4. The AIC and PRMSE support NGINAR(1) model for these data 
(see Table 13). From Table 13, it can be observed that the forecast accuracy measures are close to each other. However, the AIC criterion supports the NGINAR(1) model and hence the same has been used for forecasting. Table 14 gives the point estimates of the probabilities and Table 15 gives the 95\%confidence intervals for the forecast probability. From Table 14, we see that the transition from 1 to 0 have probabilities increasing from 0.579 to 0.685 , for steps 1 to 5 , whereas, these probabilities are decreasing for all other states from 1, when lag increases. Here, we can see that the probability of getting zero as next forecast lies between 0.537 and 0.621 , probability of getting one as next forecast lies between 0.261 and 0.292 with $95 \%$ confidence. Similar pattern appears for other steps as well. It can be seen from Table 15 that there is less chance of having value more than 1 in future, and the epidemic will be completely under control. HPP intervals (80\%), predicted mean, median and mode are given in Table 16. It can be found that the median and mode of the k- step ahead probability distribution are very good forecasts. All the actual counts are within the interval $\left(X_{L}, X_{U}\right)$.

Table 12: Descriptive statistics of Measles data

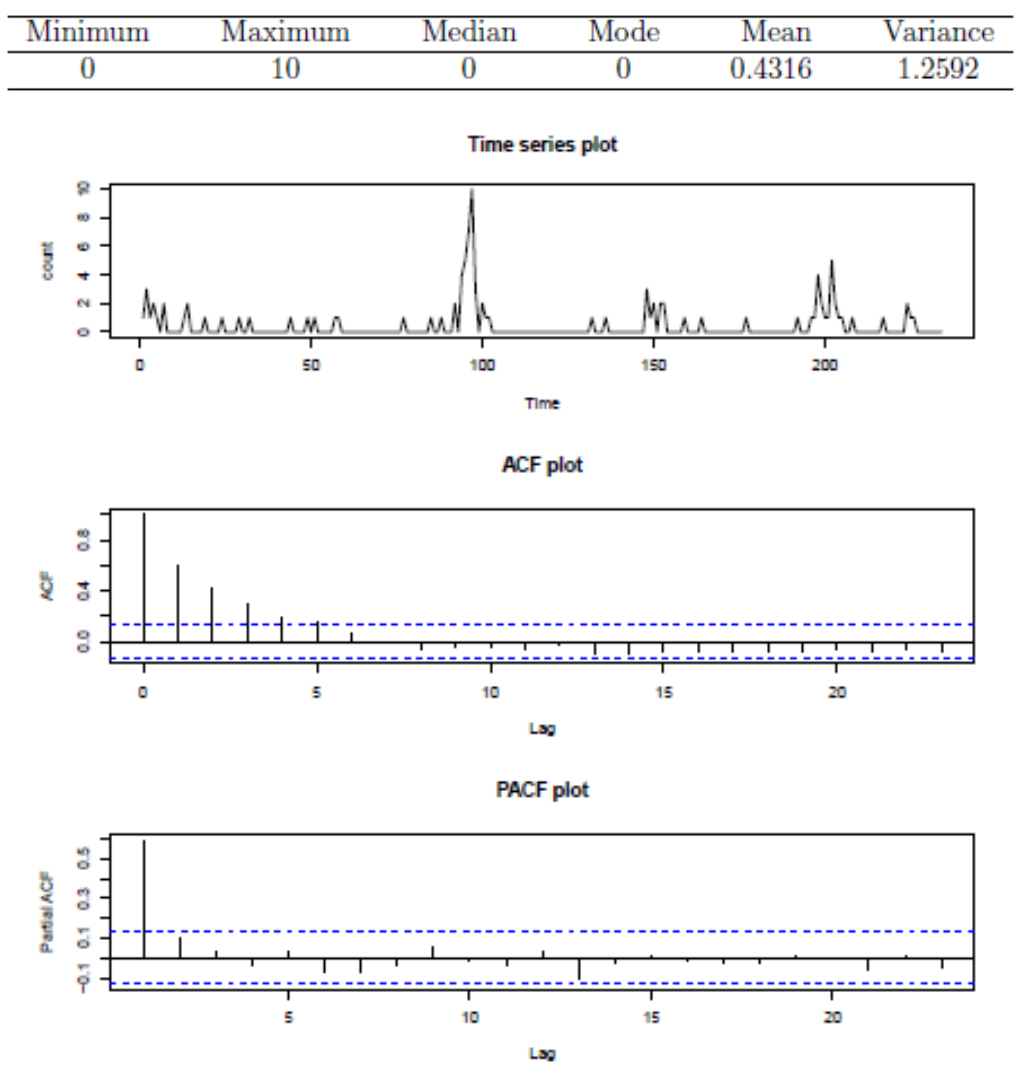

Figure 4: Time series, ACF and PACF plots of Measles data 
Table 13: Predictive analysis of Measles data

\begin{tabular}{|c|c|c|c|c|c|c|c|}
\hline Model & Estimates & AIC & $k$-step & PRMSE & PMAE & $\begin{array}{l}\text { PTP med } \\
\text { (Median) }\end{array}$ & $\begin{array}{l}\text { PTP mode } \\
\text { (Mode) }\end{array}$ \\
\hline NGINAR(1) & $\begin{array}{l}\hat{\alpha}=0.3141 \\
\hat{\mu}=0.4580\end{array}$ & 326.68 & $\begin{array}{l}1 \\
2 \\
3 \\
4 \\
5 \\
\end{array}$ & $\begin{array}{l}0.503 \\
0.536 \\
0.546 \\
0.549 \\
0.550 \\
\end{array}$ & $\begin{array}{l}0.200 \\
0.206 \\
0.214 \\
0.222 \\
0.192 \\
\end{array}$ & $\begin{array}{l}83.33 \\
82.76 \\
82.14 \\
81.48 \\
84.62 \\
\end{array}$ & $\begin{array}{l}80.00 \\
82.76 \\
82.14 \\
82.14 \\
84.62 \\
\end{array}$ \\
\hline GINAR(1) & $\begin{array}{l}\hat{\phi}=0.3521 \\
\hat{\theta}=0.3296\end{array}$ & 333.20 & $\begin{array}{l}1 \\
2 \\
3 \\
4 \\
5\end{array}$ & $\begin{array}{l}0.510 \\
0.548 \\
0.560 \\
0.564 \\
0.567\end{array}$ & $\begin{array}{l}0.200 \\
0.214 \\
0.214 \\
0.222 \\
0.192\end{array}$ & $\begin{array}{l}82.76 \\
82.14 \\
82.14 \\
81.48 \\
84.62\end{array}$ & $\begin{array}{l}82.76 \\
82.14 \\
82.14 \\
82.14 \\
84.62\end{array}$ \\
\hline
\end{tabular}

Table 14: Forecast probabilities for Measles data using NGINAR(1) model

\begin{tabular}{cccccc}
\hline & \multicolumn{5}{c}{$\mathrm{k}$} \\
\cline { 2 - 6 } & 1 & 2 & 3 & 4 & 5 \\
\hline$p_{\boldsymbol{k}}(0 \mid 1)$ & 0.579 & 0.658 & 0.678 & 0.682 & 0.685 \\
$p_{\boldsymbol{k}}(1 \mid 1)$ & 0.277 & 0.227 & 0.219 & 0.216 & 0.216 \\
$p_{\boldsymbol{k}}(2 \mid 1)$ & 0.099 & 0.077 & 0.070 & 0.069 & 0.068 \\
$p_{\boldsymbol{k}}(3 \mid 1)$ & 0.032 & 0.025 & 0.023 & 0.022 & 0.021 \\
$p_{\boldsymbol{k}}(4 \mid 1)$ & 0.009 & 0.008 & 0.007 & 0.007 & 0.001 \\
$p_{\boldsymbol{k}}(5 \mid 1)$ & 0.003 & 0.003 & 0.002 & 0.002 & 0.002 \\
\hline
\end{tabular}

Table 15: Confidence intervals $(95 \%)$ of the forecast probabilities for Measles data using NGINAR(1) model

\begin{tabular}{cccccc}
\hline & \multicolumn{5}{c}{$k$} \\
\cline { 2 - 6 } & 1 & 2 & 3 & 4 & 5 \\
\hline & & & & \\
$p_{k}(0 \mid 1)$ & $(0.537,0.621)$ & $(0.624,0.692)$ & $(0.647,0.708)$ & $(0.653,0.711)$ & $(0.657,0.714)$ \\
$p_{k}(1 \mid 1)$ & $(0.261,0.292)$ & $(0.216,0.239)$ & $(0.208,0.229)$ & $(0.206,0.227)$ & $(0.205,0.226)$ \\
$p_{k}(2 \mid 1)$ & $(0.084,0.115)$ & $(0.065,0.088)$ & $(0.060,0.080)$ & $(0.059,0.078)$ & $(0.059,0.077)$ \\
$p_{k}(3 \mid 1)$ & $(0.024,0.039)$ & $(0.019,0.032)$ & $(0.017,0.028)$ & $(0.017,0.027)$ & $(0.016,0.026)$ \\
$p_{k}(4 \mid 1)$ & $(0.007,0.012)$ & $(0.005,0.011)$ & $(0.005,0.010)$ & $(0.005,0.009)$ & $(0.000,0.003)$ \\
$p_{k}(5 \mid 1)$ & $(0.002,0.004)$ & $(0.002,0.004)$ & $(0.001,0.003)$ & $(0.001,0.003)$ & $(0.001,0.003)$ \\
\hline
\end{tabular}

Table 16: Prediction interval $(80 \%)$ with actual and forecast values using the mean, median and the mode of the conditional distribution for Measles data using NGINAR(1) model

\begin{tabular}{cccccc}
\hline$k$ & actual & $\left(X_{L}, X_{U}\right)$ & Mean & Median & Mode \\
\hline 1 & 1 & {$[0,2)$} & 0.6282 & 0 & 0 \\
2 & 0 & {$[0,2)$} & 0.5114 & 0 & 0 \\
3 & 0 & {$[0,2)$} & 0.4747 & 0 & 0 \\
4 & 1 & {$[0,2)$} & 0.4632 & 0 & 0 \\
5 & 0 & {$[0,2)$} & 0.4596 & 0 & 0 \\
\hline
\end{tabular}

\section{Conclusion}

From the detailed study of two models and the comparison of the forecast measures, it is observed that the NGINAR(1) model performs relatively better than the GINAR(1) when the count time series data are over dispersed. Two epidemiological time series data are analyzed 
and both are found to be better modeled by NGINAR(1). As several characteristics of these two models are quite different for near lags, there is a need to select the appropriate geometric INAR model for applications, depending on the data at hand. Criteria such as AIC, PRMSE etc., will help in choosing one of the geometric INAR models. From the data sets analyzed in this paper, we found that the coherent forecasts using NGINAR(1) model are better than those from the GINAR(1) model. It is also revealed that the k-step ahead median and mode are better than the MMSE forecasts.

\section{Acknowledgment}

Manik Awale would like to acknowledge the University Grants Commission (UGC) of India for an award of Research Fellowship under the Faculty Improvement Program. T.V.Ramanathan's research was partially supported by a grant from the Department of Science \& Technology (DST), Government of India, SR/S4/MS-866/13. Authors are thankful to Dr. A. S. Kashikar for some fruitful discussions they had with her. Authors are thankful to the anonymous referee and the chief editor for their comments and suggestions.

\section{References}

[1] Al-osh, M. A. \& Alzaid, A. A. (1987). First order integer-valued autoregres- sive (INAR(1)) processes. Journal of Time Series Analysis, 8, 261-275.

[2] Bu, R. \& McCabe, B. P. M. (2008). Model selecton, estimation and fore- casting in INAR(p) models: A likelihood-based Markov chain approach. International Journal of Forecasting, 24, 151-162.

[3] Ferrell, P. J., MacGibbon, B. \& Tomberlin, T. J. (2007). A hierarchical Bayes approach to estimation and prediction for time series of counts. Brazilian Journal of Probability and Statistics, 21, 187-202.

[4] Freeland, R. K. \& McCabe, B. P. M. (2004). Forecasting discrete low count time series. International Journal of Forecasting, 20, 427-434.

[5] Jazi, M. A., Jones, G. \& Lai, C. D. (2012). First-order integer valued AR processes with zero inflated Poisson innovations. Journal of Time Series Analysis, 33(6), 954-963.

[6] Jung, R. C. \& Tremayne, A. R. (2006). Coherent forecasting in integer time series models. I 22, 223-238.

[7] Kashikar, A. S., Rohan, N. \& Ramanathan, T. V. (2013). Integer autoregres- sive models with structural breaks. Journal of Applied Statistics, 40(12), 2653-2669.

[8] Kim, H. Y. \& Park, Y. (2010). Coherent forecasting in binomial AR(p) model. Communications of the Korean Statistical Society, 17(1), 27-37.

[9] Lai, D. (2005). Monitoring the SARS epidemic in China: A time series analysis. Journal of Data Science, 3, 279-293.

[10]Maiti, R. \& Biswas, A. (2015). Coherent forecasting for over-dispersed time series of counts data. Brazilian Journal of Probability and Statistics, 29(4), 747-766.

[11]Maiti, R., Biswas, A. \& Das, S. (2015). Coherent forecasting for count time series using Box-Jenkins's $A R(p)$ model. Statistica Neerlandica, 70(2), 123-145.

[12] McKenzie, E. (1986). Autoregressive moving-average processes with negative- binomial and geometric marginal distributions. Advances in Applied Prob-ability, 18, 679-705.

[13] Ngatchou-Wandji, J. \& Paris, C. (2011). On the zero-inflated count models with application to modelling annual trends in incidences of some occupa- tional allergic diseases in France. Journal of Data Science, 9, 639-659. 
[14] Özmen, İ \& Famoye, F. (2007). Count regression models with an applica- tion to zoological data containing structural zeros. Journal of Data Science, 5, 491-502.

[15] Osisiogu, U. A. \& Nwosu, C. A. (2015). Predicting Future CD4 Cell Counts of HIV/AIDS Patients by Non-Stationary Markov Chain: A Case Study of Anambra State. Journal of Data Science, 12, 95-114.

[16]Ristić, M. M., Bakouch, H. S. \& Nastić, A. S. (2009). A new geometric first order integervalued autoregressive (NGINAR(1)) process. Journal of Statistical Planning and Inference, 139, 2218-2226.

[17] Silva, N., Pereira, I. \& Silva, M. E. (2009). Forecasting in INAR(1) model. REVSTAT-Statistical Journal, 7(1), 119-134.

[18] Stuetal, F. W. \& Van Harn, K. (1979). Discrete analogues of self-decomposability and stability. Annals of Probability, 7, 893-899.

[19]Zeger, S. L. (1988). A regression model for time series of counts. Biometrika, 75(4), 621-629.

\author{
Manik Awale \\ Department of Statistics \\ Savitribai Phule Pune University \\ Pune 411007, India \\ T. V. Ramanathan \\ Department of Statistics \\ Savitribai Phule Pune University \\ Pune 411007, India \\ Mohan Kale \\ Department of Statistics \\ Savitribai Phule Pune University \\ Pune 411007, India
}

